\title{
A Passive Harmonic Tag for Humidity Sensing
}

\author{
Antonio Lazaro, Ramon Villarino, and David Girbau \\ Department of Electronics, Electrical and Automatics Engineering, Universitat Rovira $i$ Virgili, \\ Avinguda Paisos Catalans 26, 43007 Tarragona, Spain \\ Correspondence should be addressed to Antonio Lazaro; antonioramon.lazaro@urv.cat
}

Received 18 March 2014; Accepted 3 July 2014; Published 23 July 2014

Academic Editor: Ananda Sanagavarapu Mohan

Copyright (C) 2014 Antonio Lazaro et al. This is an open access article distributed under the Creative Commons Attribution License, which permits unrestricted use, distribution, and reproduction in any medium, provided the original work is properly cited.

This paper describes a passive harmonic tag for radio frequency identification (RFID) and wireless sensor applications. The tag uses a dual polarized UHF patch antenna as an input antenna. One of the outputs is connected to a frequency doubler, which consists of a Schottky diode with its output connected to a patch tuned at twice the input frequency. The other output of the input antenna feeds a DC power harvested converter that drives an oscillator which modulates its output signal by controlling the bias point of the Schottky diode. The antenna's output is also used as a humidity sensor. To achieve this, the antenna is loaded with an interdigital capacitor with humidity-dependent capacitance. The antenna is consequently detuned when humidity varies, and therefore the second harmonic power is received. The tag is manufactured using standard fiberglass substrate. The basic theory of harmonic tag operation is described and compared with the standard backscattering approach. Experimental results with a proof of concept using commercial components are presented.

\section{Introduction}

Passive RFID systems have experienced tremendous growth in recent years [1]. A passive RFID system operates as follows (see Figure 1). The RFID reader transmits a modulated RF signal to the RFID tag. It consists of an antenna and an integrated circuit (IC). The IC receives power from the antenna and responds by varying its input impedance and thus modulating the backscattered signal. In these systems, the continuous-wave $(\mathrm{CW})$ signal that powers up the passive tags is inevitably coupled to the receiver input as a strong self-interference, which presents a major challenge to the reader's receiver design. Several cancellation techniques to overcome this problem have been proposed $[2,3]$. An alternative approach based on harmonic radar can be used to avoid it. This consists of a frequency multiplier that generates a harmonic or subharmonic of the interrogation signal [4-9], providing a unique response signal among the leakages from the transmitter. Harmonic radars have been used to track insects for several years [6] using maritime radar based technologies at X-band fundamental frequencies. The transponder typically consists of a simple, lightweight antenna structure directly matched to a Schottky diode. In this application, it is possible to detect the insect at long distances within an environment with strong clutter. This feature is exploited to find avalanche victims [4, 10]. A commercial avalanche detector is available from RECCO [10] and it uses $917 \mathrm{MHz}$ fundamental frequency to achieve very good ground penetration capability. Depending on the final application, transponders that operate at different frequency ranges have been presented in the literature. UHF and L-band CW hand-held harmonic radars were used to track ground insects $[11,12]$. Other transponders have been designed at 2.45 or $5.8 \mathrm{GHz}[13,14]$. A millimeter wave transponder based on a nonlinear transmission line (NTL) has been reported in [7] with a read range less than $1 \mathrm{~m}$.

Potential applications of passive RFID technology require its functionality to be increased by adding sensing capability to the tags. These could be used to sense different environmental conditions, that is, temperature and humidity. Some commercially available sensors are usually based on active tags which make them quite expensive. Passive or semipassive tags are thus a preferred option for low-cost sensor applications [15]. Consequently, a great interest to develop passive UHF sensors has arisen in the literature. These tags are often based on detuning the antenna by the 


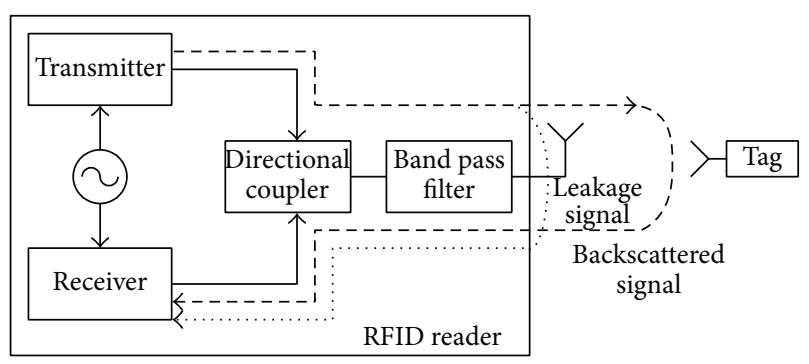

FIGURE 1: Block diagram of a UHF passive system.

sensor. Hence, both the required power to turn-on the tag and the received power depend on the physical magnitude to be read [16-18].

Harmonic scattering from passive UHF RFID tags has been used to characterize the radiation pattern diagram of the tag [19]. In a recent work [20], the residual harmonic signal from commercial UHF tags has been investigated, and it is proposed as useful tool for the development of future sensors. These works show that standard UHF tags generate backscattered harmonics. However, since the antenna is matched at the fundamental frequency, it filters these components. Therefore, the backscattered power of the harmonics is too low. In this sense, standard tags can be considered as harmonic tags due to nonlinear behaviour. One practical problem when a harmonic sensor is designed is the selection of the working frequency. In order to enhance the nonlinearities, high power should be used to achieve a great read range of meters; thus, UHF RFID band is a good candidate. However, the second harmonic can fall within other band dedicated to other services that can be interfered by the backscattered harmonics near the reader. At a few meters, the harmonic level is small, and therefore the interference will be small too and comparable to the level of harmonics in conventional UHF RFID systems. In any case, there is a lack of regulation about the frequency band for harmonic radars. However, sensors based on harmonic tags have begun to be studied in the literature [21]. For instance, a temperature harmonic tag has been proposed in the literature working at $1.5 \mathrm{GHz}$ fundamental frequency [22].

In this work, a proof of concept for a novel humidity sensor based on harmonic tag working at UHF RFID band is presented. An input rectenna is used to power a lowfre-quency oscillator that modulates the frequency doubler based on a Schottky diode. This modulation allows increasing the read range because it reduces the problems associated with the harmonic leakage in the reader. The RF signal received by the other polarisation of the input antenna is used as input signal towards the doubler. A simple low-cost interdigital capacitor that loads the output patch antenna is used as sensor. The effective permittivity changes with the relative humidity, changing the capacitance and detuning the output antenna. The paper is structured as follows. Section 2 presents the humidity sensor tag and describes the basic theory of operation. A link-budget study is done in this section in order to study the read range and the level of harmonic. A comparison between the read range that is

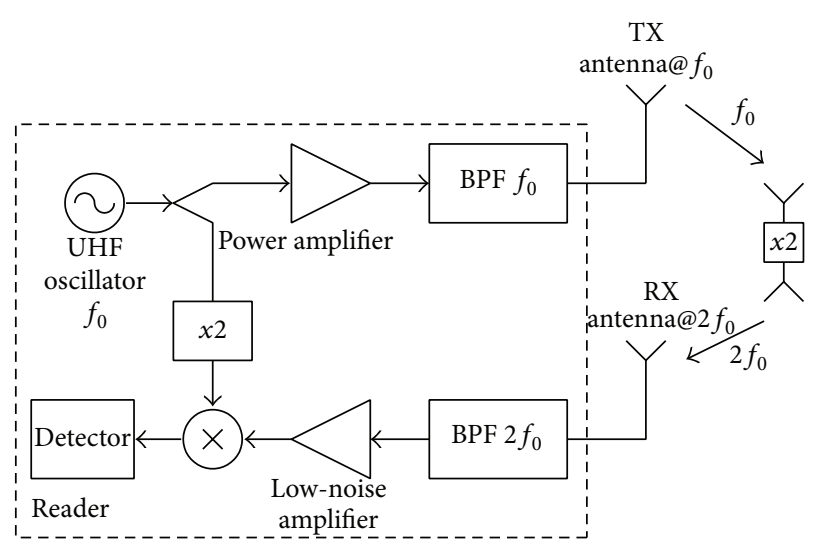

FIGURE 2: Block diagram of the system.

achievable with the proposed harmonic tag and conventional backscattering RFID systems will be studied in this section. Section 3 presents the experimental results of the system. Finally, some conclusions are drawn in Section 4.

\section{RFID System Based on Harmonic Radar}

2.1. System Description and Theory of Operation. A block diagram of the RFID system based on a harmonic tag is shown in Figure 2, comprising the reader and the tag. The tag can be observed in Figure 3. It consists of a dual-polarized patch reception $(\mathrm{Rx})$ antenna that collects the interrogation signal at a UHF frequency of $f_{0}(865-868 \mathrm{MHz})$. This antenna is connected to the transmission $(\mathrm{Tx})$ patch antenna by means of a frequency doubler. The Tx antenna is loaded with an interdigital capacitor with a capacitance dependent on the humidity so that, when the relative humidity changes, the antenna is detuned. A variation in the received power at the reader is thus obtained. The experimental results given in the next section show that despite using filters at the transmitter output, the amplitude of the leakage signal at $2 f_{0}$ is larger than the tag reflected amplitude at the second harmonic. Modulation of the tag is therefore required to exploit the receiver's sensitivity. To that end, the second port of the $\mathrm{Rx}$ antenna is connected to a RF to DC converter module, which supplies a low-frequency oscillator. This oscillator modulates the output signal at $2 f_{0}$ by controlling the bias point of the frequency doubler. In consequence, the tag answers the reader modulating the second harmonic. The reader's mixer demodulates the backscattered signal using the second harmonic of the transmitted signal as the local oscillator. The tag is manufactured using standard fiberglass substrate FR4. The $\mathrm{Rx}$ antenna is a square patch antenna $79 \mathrm{~mm}$ wide and an inset line length of $14 \mathrm{~mm}$, inset line width of $1 \mathrm{~mm}$, and an inset gap of $1 \mathrm{~mm}$. The frequency conversion in harmonic tags is generally accomplished by a Schottky diode which should have zero-bias high voltage sensitivity and a low barrier in order to maximize the conversion of small signals. In this study, the frequency doubler is based on a zero-bias Schottky diode (Avago Technologies, model HSMS-2850). The fundamental signal at $f_{0}$ is filtered using 


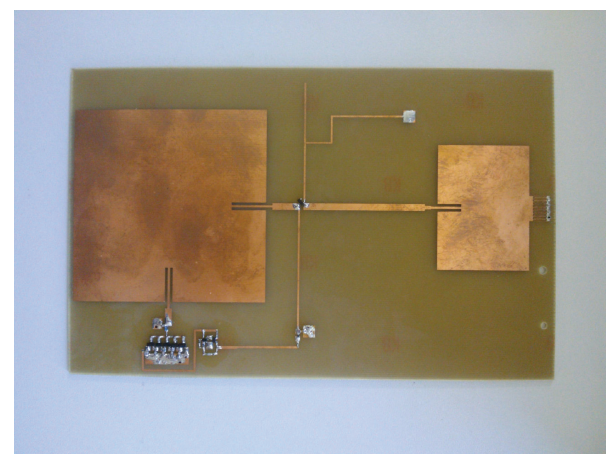

(a)

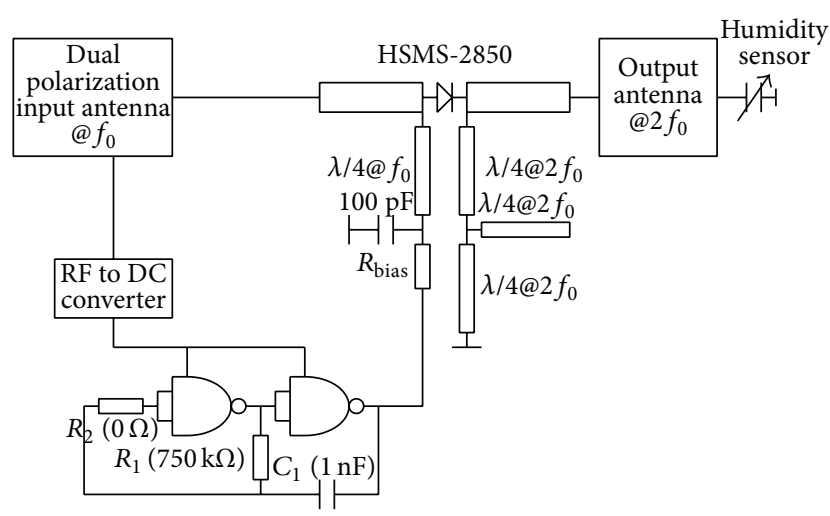

(b)

Figure 3: Photograph of the implemented tag (a) and block diagram (b).

a high pass filter implemented with a $\lambda / 4$ stub at $2 f_{0}$. The frequency doubler blocks the second harmonic at the input using a short-circuited $\lambda / 4$ stub at $f_{0}$ using a bypass capacitor. The output of doubler can be modulated by changing the diode DC bias point. To this end, a bias resistor $\left(R_{\text {bias }}\right)$ is inserted at the output of the oscillator. The end of the quarter wavelength line is connected to the ground at RF by a bypass capacitor. The high value of the resistor $(1 \mathrm{k} \Omega)$ also blocks the RF signal and limits the DC current consumption, while the DC return is allowed through the resistor. The modulating capability is shown in Figure 4. This figure shows the measured conversion loss of the designed frequency doubler at $868 \mathrm{MHz}$ for $0 \mathrm{~V}$ and a $0.5 \mathrm{~V} \mathrm{DC}$ bias. With an input power under $-10 \mathrm{dBm}$, a difference larger than $12 \mathrm{~dB}$ between the two states is observed.

The RF to DC converter is based on a diode voltage multiplier (see Figure 5) using series-connected zero-bias diodes (Avago Technologies, model HSMS-2852). Figure 6 shows the measured detected voltage as a function of input power at $868 \mathrm{MHz}$. The low-frequency oscillator is implemented with two NAND logic gates (74AUP1G00). This oscillator needs $0.45 \mathrm{~V}$ to start oscillating. The minimum input power to obtain this voltage is thus about $-19 \mathrm{dBm}$, which is close to the value achieved by commercial UHF RFID tags (e.g., Impinj Monza Gen 5 or Alien Higgs-3 ICs). The output of this oscillator is connected to the input stub of the doubler to modulate its conversion loss.

2.2. Loaded Patch Antenna as a Humidity Sensor. The Tx patch antenna is tuned to the center frequency of the second harmonic and loaded with a variable capacitor. This structure is used as a humidity sensor. The capacitor is an interdigital capacitor connected to the ground. Fiberglass is known to change its permittivity depending on the water content. A low-cost integrated humidity sensor is therefore designed using fiberglass (relative permittivity $\varepsilon_{r}=4.4$, substrate height $1.6 \mathrm{~mm}$ ). When the relative humidity increases, the surface permittivity of the substrate between the interdigital electrodes also increases. The capacitance consequently increases, and the resonant frequency of the patch antenna

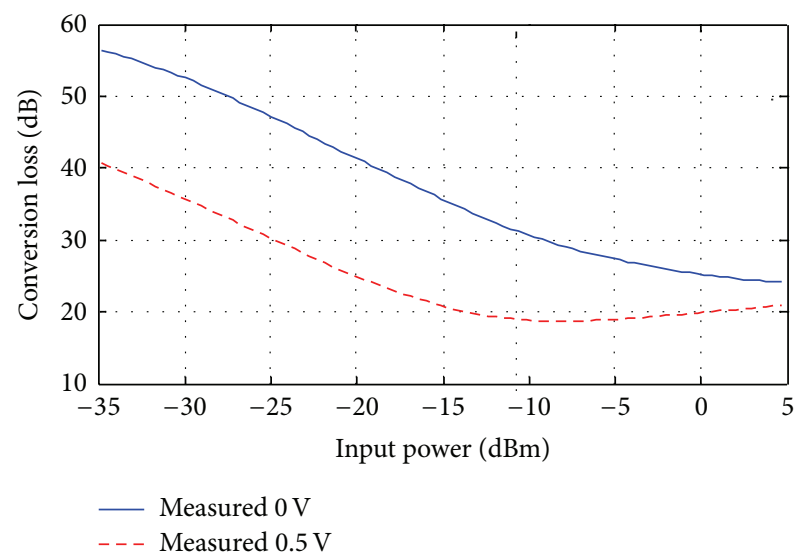

FIGURE 4: Measured conversion loss of the frequency doubler for $0 \mathrm{~V}$ and $0.5 \mathrm{~V}$ DC bias voltage at $868 \mathrm{MHz}$.

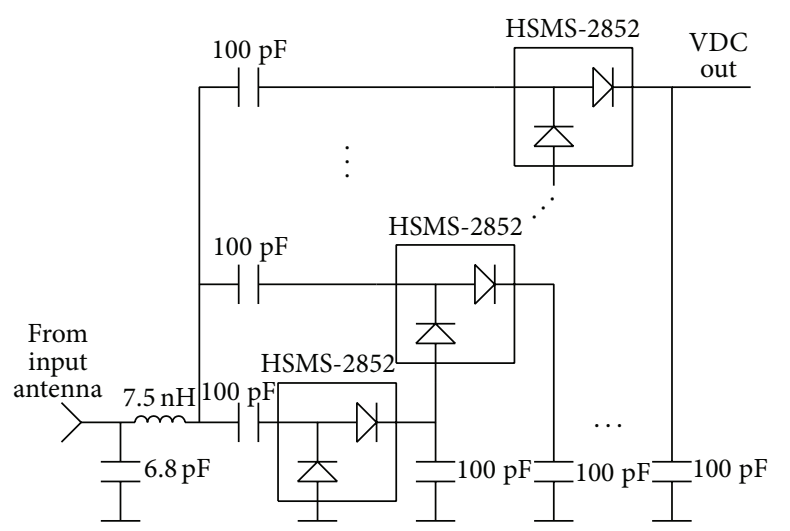

Figure 5: RF to DC converter module based on a 5-level voltage multiplier.

therefore changes. Figure 7 shows the layout of the antenna with its main dimensions.

A model based on a transmission line to understand the loaded antenna operation is shown in Figure 7. $L$ is the length 


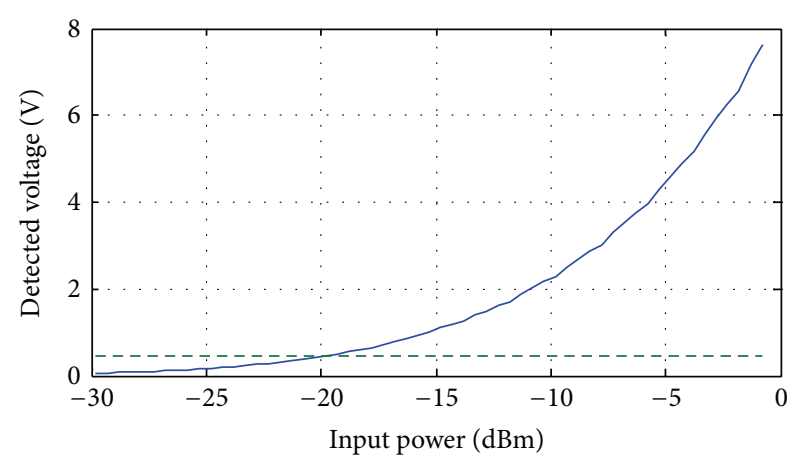

FIGURE 6: Measured detected voltage as a function of input power at $868 \mathrm{MHz}$. The dashed line shows the minimum voltage needed to power up the oscillator $(0.45 \mathrm{~V})$.

of the patch, and $\Delta L$ is the extension length in order to take into account the end capacitance of the patch. $R_{\mathrm{rad}}$ models the radiation resistance of the patch. The input match is obtained by adjusting the inset length, $L_{\text {inset }}$. From this model, it is evident that the resonant frequency of the patch can be tuned with the capacitance $\mathrm{C}$ that is a function of the humidity. The patch and input matching network are adjusted using Agilent Momentum for an interdigital capacitor with the nominal permittivity of the substrate. The patch dimensions are $W=$ $51 \mathrm{~mm}, L=37.5 \mathrm{~mm}$, and $L_{\text {inset }}=9.5 \mathrm{~mm}$ with an inset gap of $1 \mathrm{~mm}$. An 18-finger interdigital capacitor with a length of $5 \mathrm{~mm}$, a width of $0.6 \mathrm{~mm}$, and a gap between fingers of 300 microns has been designed.

Figure 8 shows the capacitance obtained from the measured reflection coefficient for different humidity percentages. As expected, the capacitance is proportional to the effective permittivity that increases with the relative humidity. Figure 9 shows the variation of capacitance and quality factor as a function of humidity.

Figure 10 compares the input reflection of the antenna loaded with the sensor and without the sensor (antenna unloaded). In the case of the antenna unloaded, the variation of the resonant frequency is small because the patch protects the substrate from the humidity. However, a small change due to the humidity in the fringing capacitance modeled with the effective length increment $\Delta L$ is observed. The variation of the resonance frequency is $3.2 \mathrm{MHz}(0.17 \%)$. Figure 10 shows the input reflection coefficient of the loaded patch antenna, which is close to $-10 \mathrm{~dB}$ for the entire relative humidity range. As the model of Figure 7 predicts, the main effect of the interdigital capacitor is a reduction of the resonance frequency. Figure 11 shows the measured resonance frequency of the loaded antenna as a function of relative humidity. The frequency range is between $1740 \mathrm{MHz}$ and $1728 \mathrm{MHz}(0.69 \%)$, which is approximately within double the frequency range of the European RFID band. A small shift between the nominal $(1730-1736 \mathrm{MHz})$ and measured resonance frequency range can be caused by the tolerance between the gaps and the lines of the interdigital capacitor in the fabrication process. Higher sensitivity can be obtained increasing the capacitance of the interdigital capacitor. These figures show that the sensor has greater sensitivity for relative humidities larger than $40-50 \%$.
As expected, the quality factor decreases with the water content due to the water losses, and the antenna suffers a small mismatch when the relative humidity approaches 100\%. The temperature dependence of the FR4 substrate causes changes on its permittivity. Consequently, the sensor must be characterized as function of the temperature. The measured sensitivity of the resonance frequency with respect to the temperature in the range between 25 and $100^{\circ} \mathrm{C}$ was $-0.847 \mathrm{MHz} / \mathrm{K}$.

2.3. Link Budget. The aim of this section is to study the performance of the harmonic radar as a function of the distance. A comparison with the conventional backscattering method using a single antenna is made for reference. For Passive RFID, the read range is limited by two factors [23]: the need for very strong power to feed the tag, limiting the reader to the tag range, and the backscattered power from the tag to respond to the reader, limiting the tag to the reader range.

The power transferred from the reader to the tag $P_{r \text {,tag }}$ under matching conditions can be calculated from the Friis equation. However, propagation in RFID environments differs from free-space conditions. An approach for predicting average power level in indoor environments for mobile communications is the dual slope model [24]. This model is derived from a two-ray path model where there are a direct ray and a reflected ray. This model assumes that, for large distances, the angle of incidence of the reflected ray approaches $90^{\circ}$, and the reflection coefficient is -1 . Physically, this corresponds to the case where the propagation path is within the first Fresnel zone boundary for a distance greater than the breaking distance, $R_{\mathrm{B} 1}$, as follows:

$$
R_{\mathrm{B} 1}=\frac{4 h_{\text {reader }} h_{\mathrm{tag}}}{\lambda_{\mathrm{in}}}
$$

where $h_{\text {reader }}$ and $h_{\text {tag }}$ are the transmitter and receiver antenna heights (reader and tag antenna heights in this case) and $\lambda_{\text {in }}$ is wavelength at frequency $f_{\text {in }}$. Using this model, $P_{r \text {,tag }}$ is given by the following expression [24]:

$$
P_{r, \mathrm{tag}}=\frac{P_{T} G_{T}}{4 \pi}\left(\frac{1}{d^{n_{1}}} \cdot \frac{1}{\left(1+d / R_{\mathrm{B} 1}\right)^{n_{\mathrm{B}}-n_{1}}}\right) A_{e, \mathrm{tag}}
$$

where the effective area of the input tag antenna is given by

$$
A_{e, \mathrm{tag}}=\frac{\lambda_{\text {in }}^{2}}{4 \pi} G_{\text {tag, in }},
$$

where $G_{\text {tag, in }}$ is the gain of the input antenna, $P_{T}$ is the transmitted power, and $A_{e, \text { tag }}$ is the effective area at frequency $f_{\text {in }}$. For distances $d \ll R_{\mathrm{B} 1}$, the path loss exponent is $n_{1}$, and, for distances $d \gg R_{\mathrm{B} 1}$, the path loss exponent is $n_{B}$. The freespace model is recovered when the antenna heights are large ( $R_{\mathrm{B} 1}$ very large) and $n_{1}$ is set to 2 . Using the flat earth model, at the limit the path loss exponent tends to $n_{B}=4$. Values of $n_{1}>2$ correspond to radio environments that include multiple path propagation effects. A typical range of $n_{1}$ in RFID environments is $2<n_{1}<2.5$ [25] and depends on the polarization and the antenna heights. 


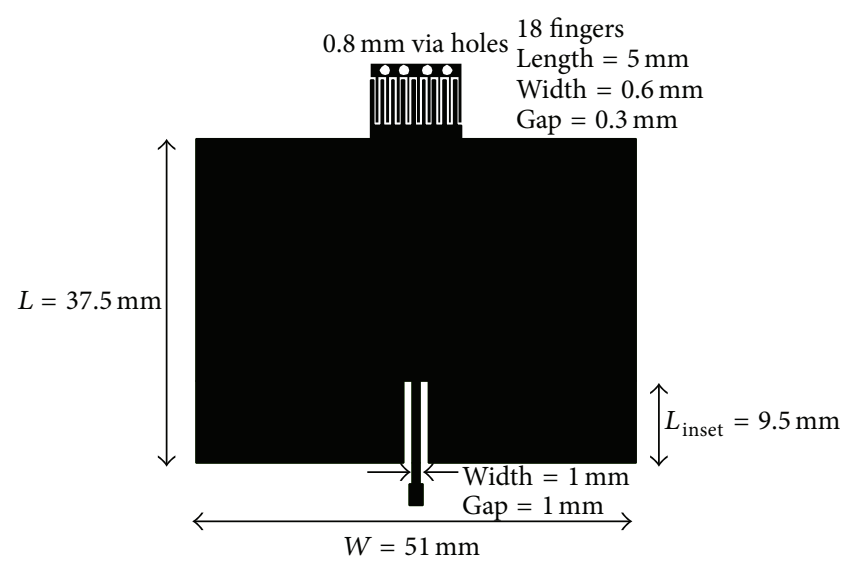

(a)

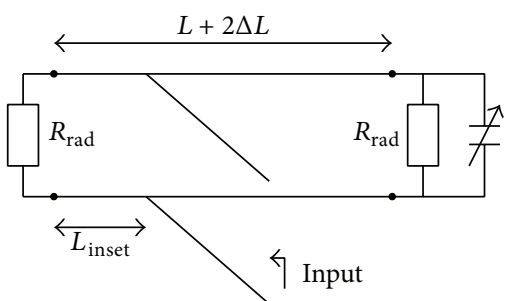

(b)

Figure 7: (a) Antenna layout. (b) Antenna loaded with a humidity sensor model.

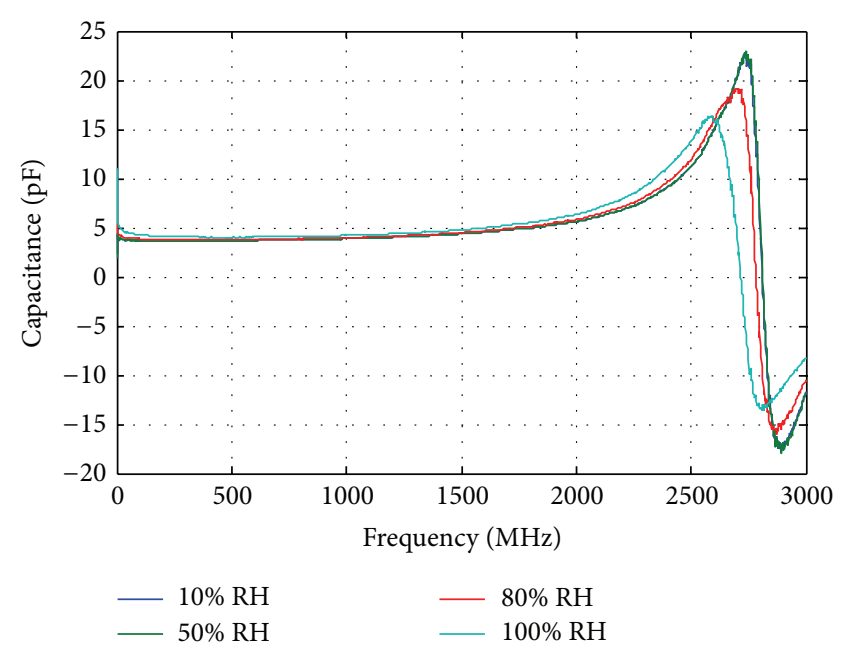

FIGURE 8: Measured capacitance as a function of the frequency for different relative humidity contents.

The power received by the tag must be greater than the tag sensitivity or the minimum power (also known as power-up threshold, $P_{\text {th }}$ ) that is needed for the rectification of the incident RF power to feed the tag and to generate the response by modulating the radar cross section. The power backscattered by the tag can be calculated using the concept of a differential cross section described in [23] and the twoslope propagation model as follows:

$$
\begin{aligned}
& P_{r, \text { reader }} \\
& =P_{r, \text { tag }} G_{\text {doubler }} \cdot \frac{G_{\text {tag,out }}}{4 \pi}\left(\frac{1}{d^{n_{2}}} \cdot \frac{1}{\left(1+d / R_{\mathrm{B} 2}\right)^{n_{B}-n_{2}}}\right) K \frac{\lambda_{\text {out }}^{2}}{4 \pi} G_{R},
\end{aligned}
$$

where $G_{\text {tag,out }}$ is the gain in the tag output antenna, $G_{R}$ is the reader receiver antenna gain and the wavelength at frequency $2 f_{\text {in }}$, and $G_{\text {doubler }}$ is the average gain of the frequency doubler. In (4), $n_{2}$ is the path loss exponent for $d \ll R_{\mathrm{B} 2}$, and the breaking distance $R_{\mathrm{B} 2}$ is calculated from (1) but using the wavelength at the frequency $f_{\text {out }}=2 f_{\text {in }}$. The classical backscattering case can be recovered setting $\lambda_{\text {in }}=\lambda_{\text {out }}$, $G_{\text {tag,in }}=G_{\text {tag,out }}$, and $G_{\text {doubler }}=1$.

In (4), $K$ is the modulation loss factor [23]. In conventional backscattering systems, $K$ can be estimated as

$$
K=\frac{1}{4}\left|\rho_{1}-\rho_{2}\right|^{2}
$$

The factor $K$ for a BPSK modulation with a $50 \%$ duty cycle is $1 / 4$ and $\rho_{1}$ and $\rho_{2}$ are the complex reflection coefficients for the two tag states. In passive UHF tags, the typical choice of modulating states is matched and short, which results in a $-6 \mathrm{~dB}$ modulation loss. In practice, the modulation loss is lower because the reflection coefficient module does not reach the unit and depends on the incident power. Typical values of -12 to $-23 \mathrm{~dB}$ were experimentally found [23]. A modulation loss of $-12 \mathrm{~dB}$ is considered in the simulations for both systems.

In the case of the harmonic tag, the output amplitude at the output frequency is modulated by a $50 \%$ duty cycle square waveform. The time domain signal can be approximated by

$$
x(t)=\left(A_{1}+\left(A_{2}-A_{1}\right) \sum_{n=1}^{\infty} \prod\left(\frac{t-n T}{T / 2}\right)\right) \cos \left(2 \pi f_{\text {out }} t\right),
$$

where $T$ is the period of the modulating signal and $A_{1}$ and $A_{2}$ are the amplitudes for the two states which are proportional to the square of conversion loss of the doubler given by Figure 4 . From the Fourier transform of (6), the modulation factor loss $K$ in a harmonic radar case can be obtained as

$$
K=\frac{2}{\pi^{2}}\left(\frac{A_{2}-A_{1}}{A_{1}+A_{2}}\right)^{2}
$$

Figure 12 shows the calculated modulation factor $K$ using (7) and the conversion loss of Figure 4 . Values between -12 and $-10 \mathrm{~dB}$ are observed for input powers under $-10 \mathrm{dBm}$. 


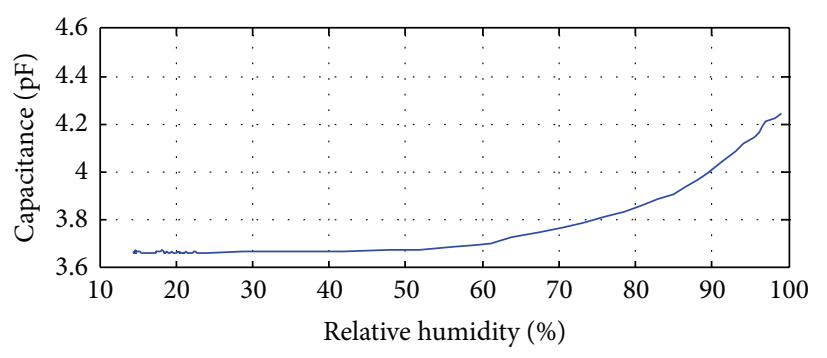

(a)

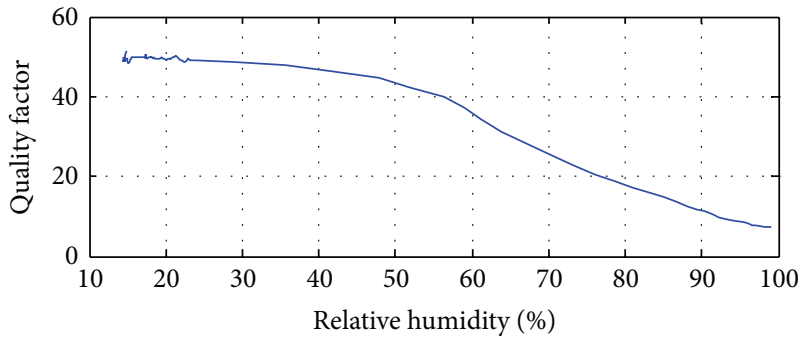

(b)

FIgURE 9: Measured capacitance (a) and quality factor (b) as a function of relative humidity at $868 \mathrm{MHz}$.

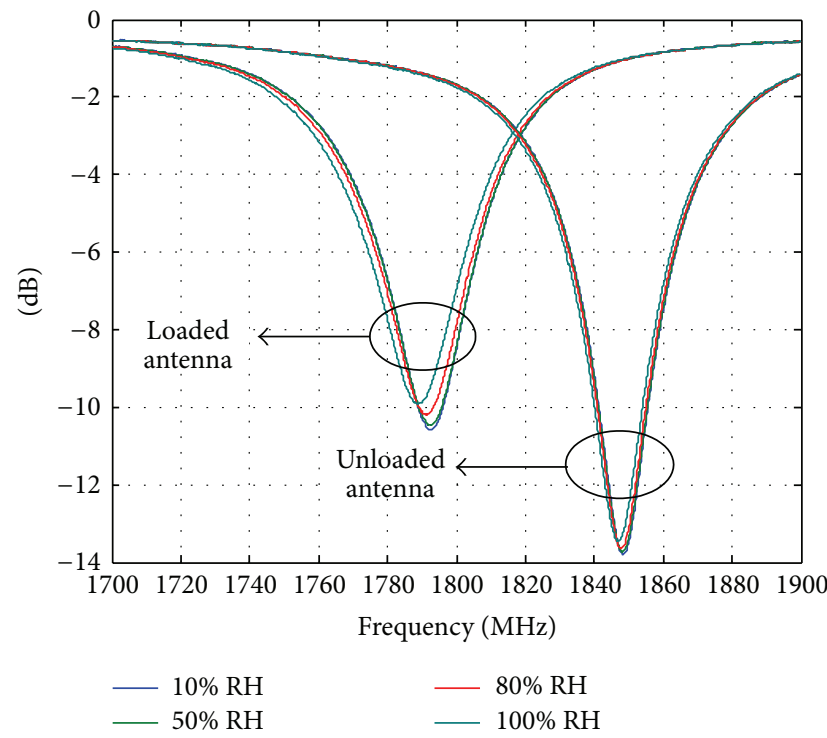

Figure 10: Measured input reflection coefficient as a function of frequency for patch antenna without the sensor and the loaded patch antenna with the sensor.

Equation (2) is only valid under matched conditions. This is approximately true for the harmonic tag because the impedance that loads the antenna does not change much between states. However, in a conventional backscattering tag, the average mismatch coefficient should be taken into account. Consider

$$
M=1-\frac{\left|\rho_{1}\right|^{2}+\left|\rho_{2}\right|^{2}}{2} .
$$

Under the previous conditions (matching and short states), $M$ is in the order of 0.5 . A value of $M=0.5$ is chosen in the simulation for the two systems.

In order to study the read range limiting factors, the threshold power and reader sensitivity for both systems are required. For instance, for the commercial tag Impinj Monza Gen 5, the threshold power $P_{\text {th }}$ is about $-20 \mathrm{dBm}$. For a commercial UHF reader, a signal-to-noise ratio (SNR) of $11.6 \mathrm{~dB}$ is required to guarantee $0.001 \%$ of BER [26]. The typical reader sensitivity for a commercial reader is limited by the leakage power to approximately $-70 \mathrm{dBm}$

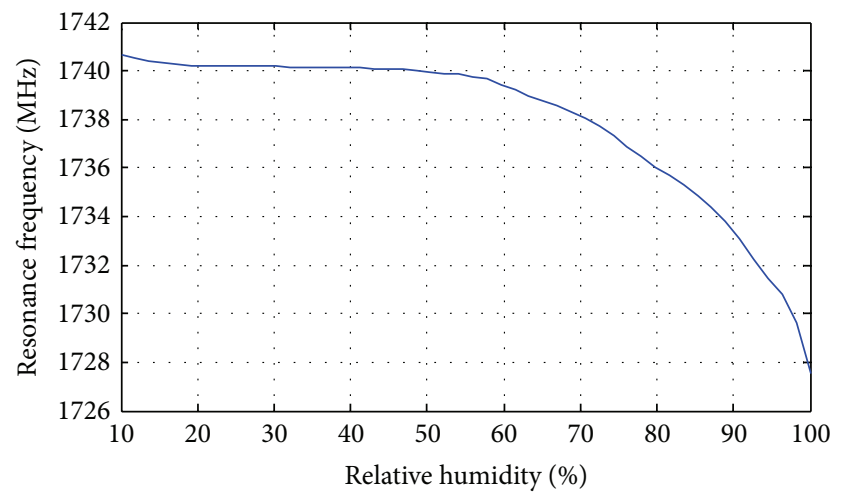

FIGURE 11: Measured resonance frequency as a function of relative humidity.

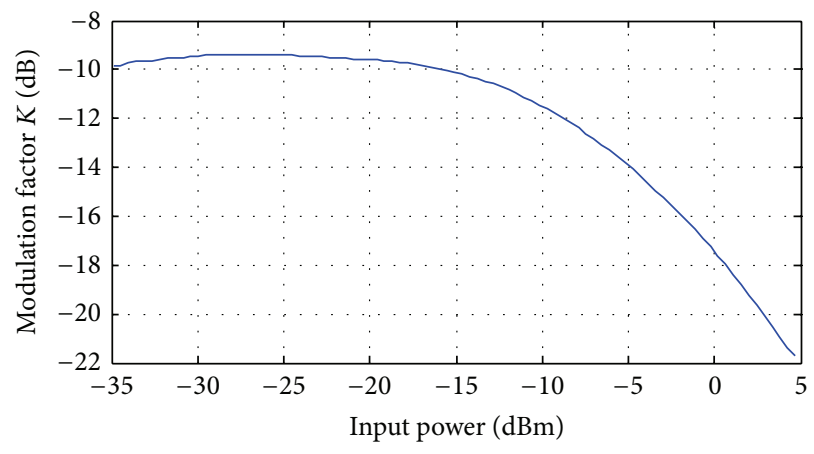

FIGURE 12: Calculated modulation factor $K$ as a function of the input power for the designed harmonic tag.

$[23,26]$. The required receiver noise floor for commercial readers is therefore $-81.6 \mathrm{dBm}[26]$. The receiver sensitivity of harmonic radar can be improved because the leakage signal at the transmitted frequency can be easily filtered in the receiver, and the leakage at the second harmonic can be reduced by filtering the signal at the output of the transmitter. The noise floor can thus be reduced below $-138 \mathrm{dBm}$, using a receiver with a $6 \mathrm{~dB}$ of noise figure and a spectrum analyzer resolution bandwidth of $1 \mathrm{KHz}$. The phase noise is consequently the most limiting factor. The typical phase noise of $-120 \mathrm{dBc} / \mathrm{Hz}$ at $100 \mathrm{kHz}$ offset can be obtained using commercial UHF phase-locked oscillators. A reader sensitivity better than $-120 \mathrm{dBm}$ can thereby be achieved. 


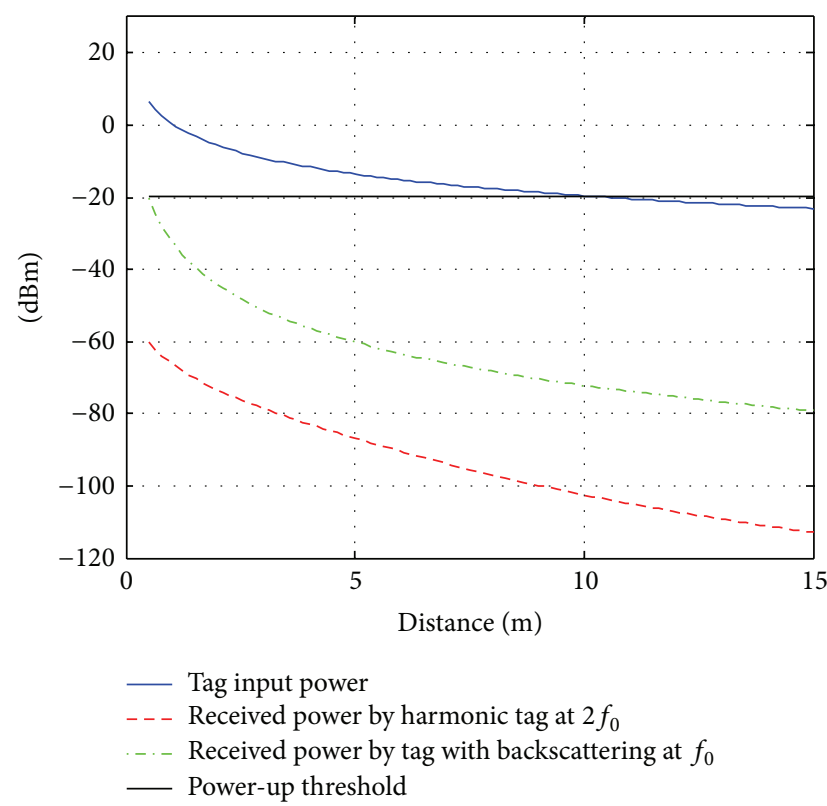

(a)

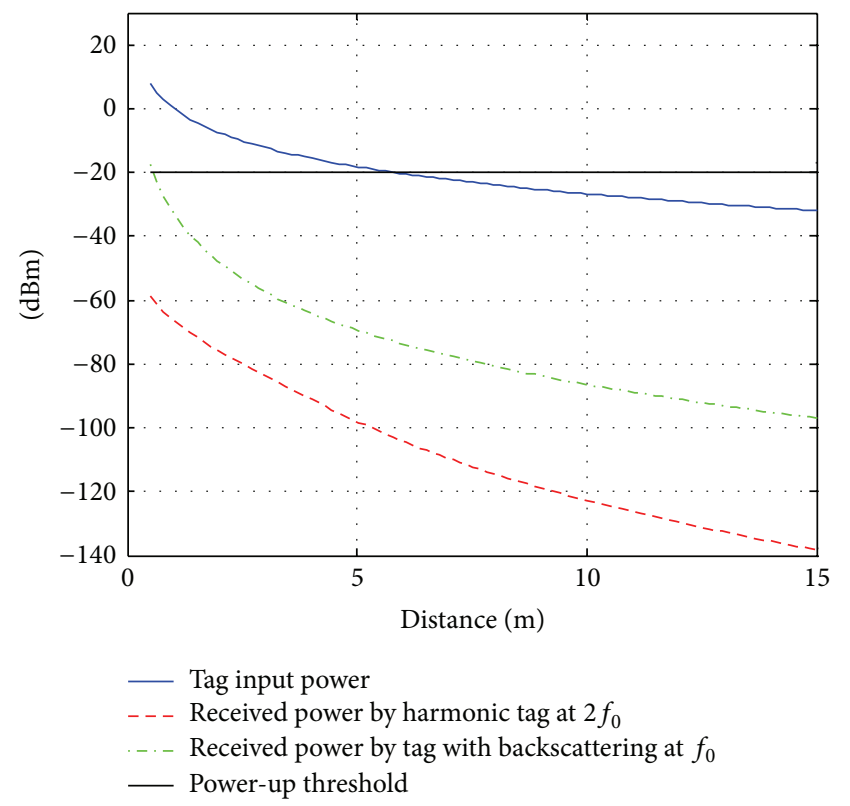

(b)

FIGURE 13: Simulated tag input power and received power by a harmonic tag and conventional tag using a free-space model (a) and using a two-slope model with $n_{1}=n_{2}=2.5$, and $h_{\text {reader }}=h_{\text {tag }}=1.5 \mathrm{~m}$. (b) The power-up threshold $(-20 \mathrm{dBm})$ is also shown.

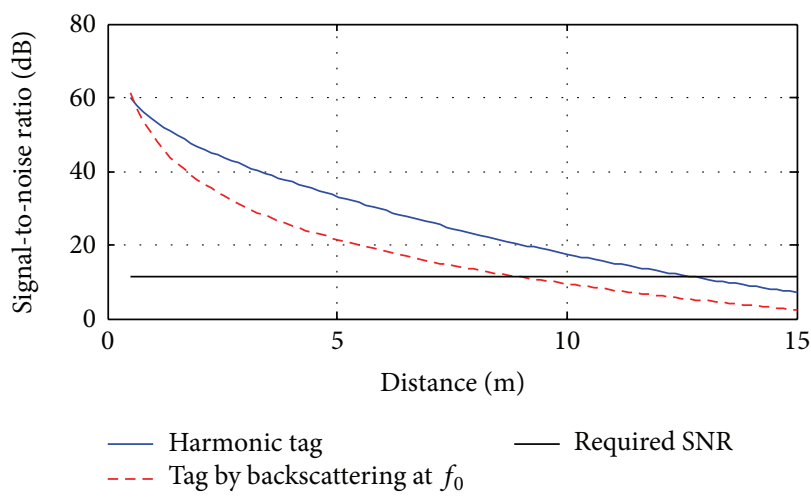

(a)

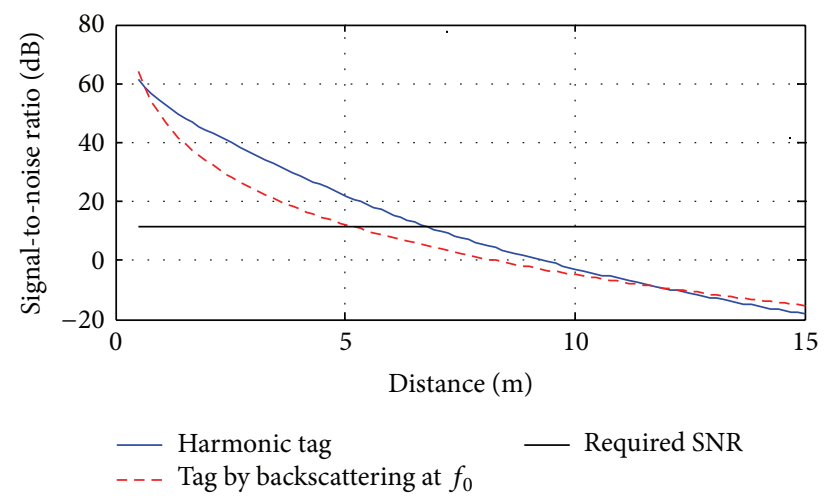

(b)

FIGURE 14: Simulated signal-to-noise ratio by a harmonic tag and convention tag by backscattering at $f_{0}$ using a free-space model (a) and using a two-slope model with $n_{1}=n_{2}=2.5$, and $h_{\text {reader }}=h_{\text {tag }}=1.5 \mathrm{~m}$. The required SNR limit of $11.6 \mathrm{~dB}$ for a $0.001 \%$ of BER is also shown.

The following figures show a comparison between the two systems, based on the conventional backscattering method and harmonic radar. The simulation assumes that the transmitted power complies with the European RFID band regulation where the equivalent isotropic radiated power (EIRP) is limited to $33 \mathrm{dBm}$. This value is taken into account in the simulations of both systems. Figure 13(a) shows the power received at the tag and the power received at the reader in both systems as a function of the distance, using a free-space model $\left(n_{1}=n_{2}=2, R_{\mathrm{B} 1}=R_{\mathrm{B} 2}=\infty\right)$, whereas Figure 13(b) shows the simulation using a more realistic propagation model $\left(n_{1}=n_{2}=2.5\right.$, and the antenna heights $h_{\text {reader }}=h_{\text {tag }}=$ $1.5 \mathrm{~m}$ ). Figure 14 shows the signal-to-noise ratio (SNR) in both systems as a function of distance for the two propagation models. The gains of the reader antennas are assumed to be equal to $6 \mathrm{~dB}$, and the antenna gain corresponds to the simulated gain for the patch antennas used in the harmonic tag design, $1.6 \mathrm{~dB}$ for the input patch and $2.6 \mathrm{~dB}$ for the output patch. In the case of the tag by backscattering, the antenna gain is assumed to be the same as the input antenna for the harmonic tag. A power-up threshold of $-20 \mathrm{dBm}$ is considered in the simulations.

Some conclusions can be obtained from these figures. First, the maximum range considering only the reader-totag communication is the same in the two RFID systems if the same antenna and mismatch coefficient are taken into account. However, whereas the read range in conventional systems is limited by the tag-to-reader communication, in the harmonic radar it is limited by the reader-to-tag radiolink. Depending on the radiopropagation model considered, 

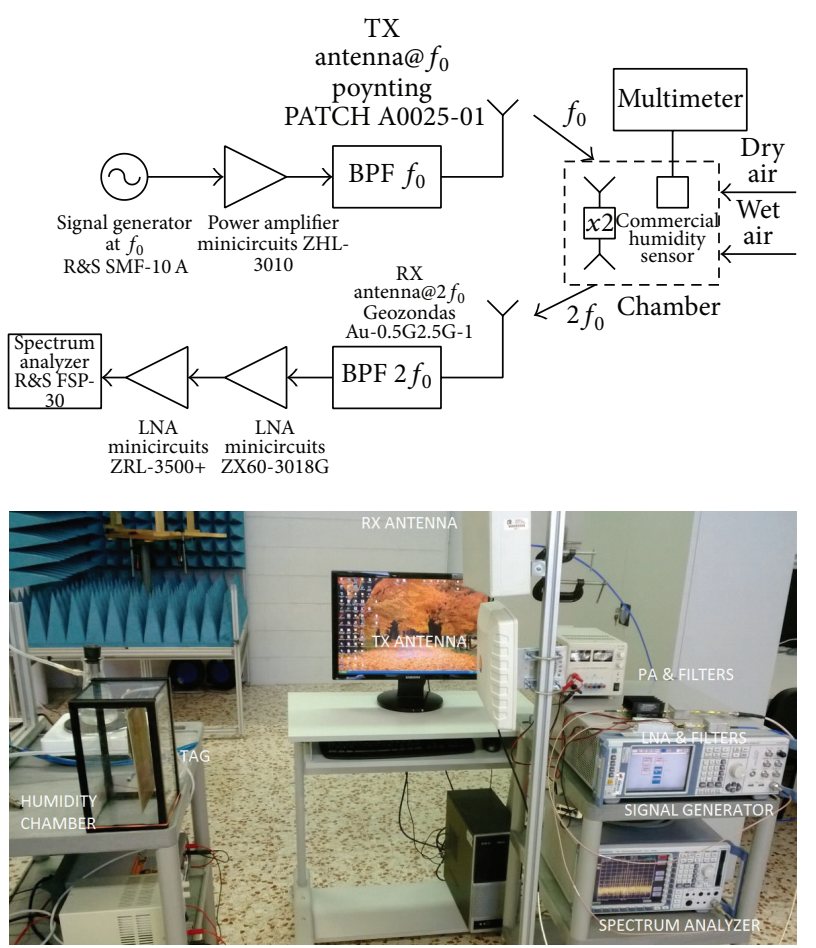

FIGURE 15: Block diagram and photography of the experimental setup used.

the simulated harmonic tag read range varies between $10 \mathrm{~m}$ (free space) and $5.8 \mathrm{~m}$ (using a two-slope model with $n_{1}=$ $n_{2}=2.5$ and $h_{\text {reader }}=h_{\text {tag }}=1.5 \mathrm{~m}$ ). In conventional RFID systems, the simulated read range varies between $8.8 \mathrm{~m}$ (free space) and $5 \mathrm{~m}$ (using a two-slope model with $n_{1}=n_{2}=2.5$ and $h_{\text {reader }}=h_{\text {tag }}=1.5 \mathrm{~m}$ ). A better signal-to-noise ratio (SNR) can thus be achieved using harmonic radars because of their greater sensitivity, although the received power at the reader is lower than in conventional RFID systems due to the tag conversion loss. This SNR improvement is greater than $10 \mathrm{~dB}$ at a distance of $5 \mathrm{~m}$. Harmonic RFID systems are consequently more robust in front multipath fading effects because they allow larger fading margins than conventional RFID systems.

\section{Experimental Results}

Some experiments were performed in order to validate the prototype designed. The experimental setup is similar to the one proposed in Figure 2 and is described in Figure 15. The oscillator is implemented using a Rohde \& Schwarz SMF-100A signal generator. Its output signal is amplified by a Minicircuits ZHL-3010 power amplifier. A notch filter is connected between the power amplifier and the UHF antenna in order to reduce the spurious emissions at the second harmonic. The commercial UHF RFID antenna model PATCH A0025-01 from Poynting, with circular polarisation and a gain of $6 \mathrm{~dB}$, is used. The level of the signal generator is adjusted to obtain an EIRP of $33 \mathrm{dBm}$ at the antenna's output. A Rohde \& Schwarz FSP-30 spectrum analyser model is

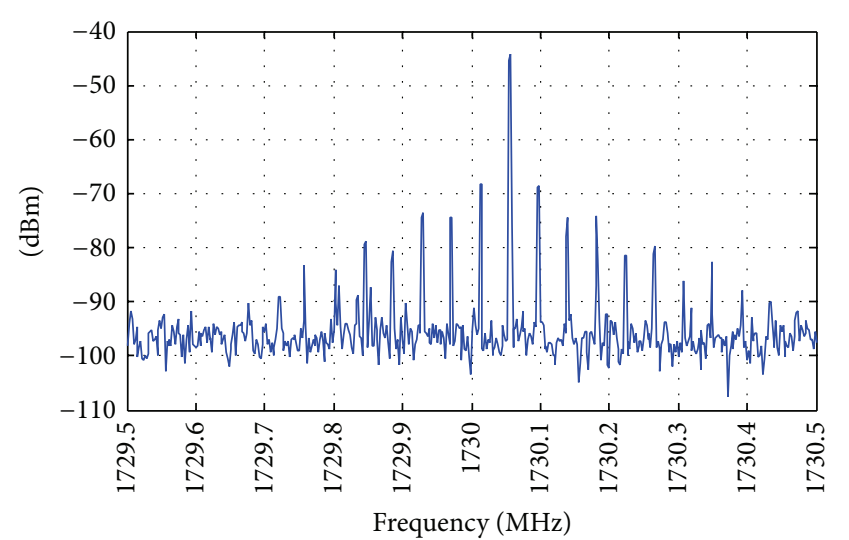

FIGURE 16: Measured spectrum at a distance of $7 \mathrm{~m}$ between tag and reader. The antenna height is $1.5 \mathrm{~m}$ and the input frequency is $865 \mathrm{MHz}$. The resolution bandwidth of the spectrum analyzer is set to $1 \mathrm{KHz}$.

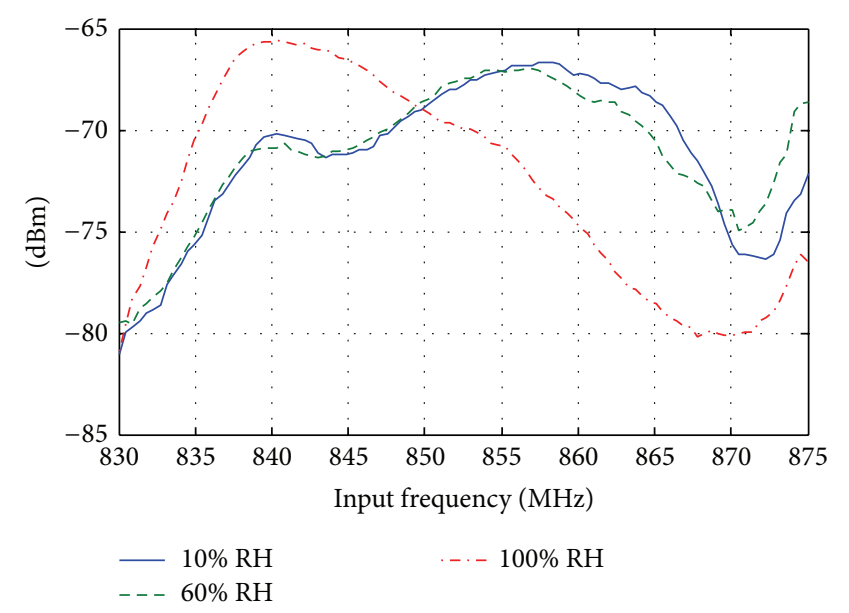

FIGURE 17: Measured variation of the received power as a function of the input frequency for different relative humidity contents. The tag-to-reader distance was $1.5 \mathrm{~m}$.

used as receiver. A low-noise amplifier (LNA) composed of the cascade connection of a ZX60-3018G-S+ and ZRL$3500+$ from Minicircuits is used to reduce the overall noise factor. A band pass filter centered at the second harmonic is connected to the input of the amplifier to reduce the level of the fundamental UHF signal and is coupled between the transmitter and receiver antennas to prevent the saturation of the amplifiers. The overall noise factor is approximately $5 \mathrm{~dB}$. A broadband antenna model AU-0.5G2.5G-1 from Geozondas with a gain of $6 \mathrm{dBi}$ at $1.7 \mathrm{GHz}$ is used as a receiver antenna. A custom climatic chamber is made. A wet air flow is used to increase the relative humidity, while a dry air flow is injected to reduce the humidity. The chamber is made with glass to prevent excessive attenuation of radiofrequency signals. A commercial humidity sensor (model HIH-5030 from Honeywell) is used as a reference to measure the relative humidity.

In order to check the read range predicted by the link budget, Figure 16 shows the spectrum at the reader-to-tag distance of $7 \mathrm{~m}$ in an indoor environment. This distance is within 


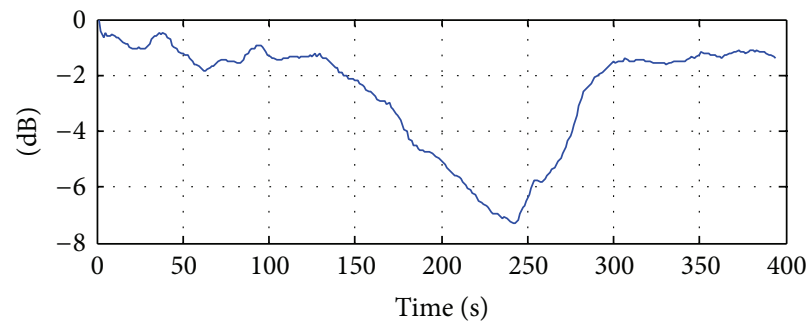

(a)

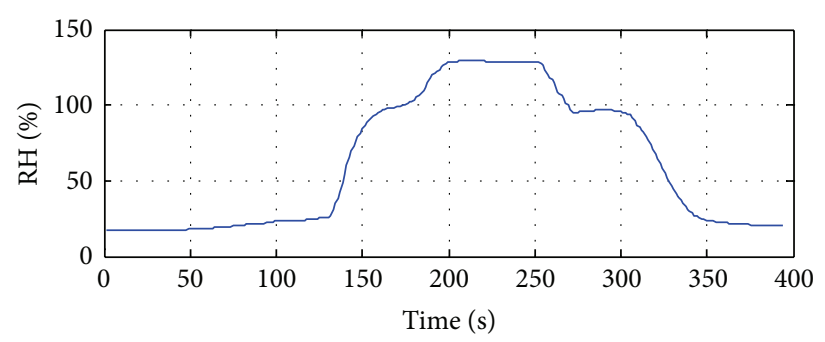

(b)

FIGURE 18: Measured variation of the received power (a) and relative humidity measurement of a commercial sensor located near the tag. Input frequency of $865 \mathrm{MHz}$ and tag-to-reader distance of $1.5 \mathrm{~m}$.

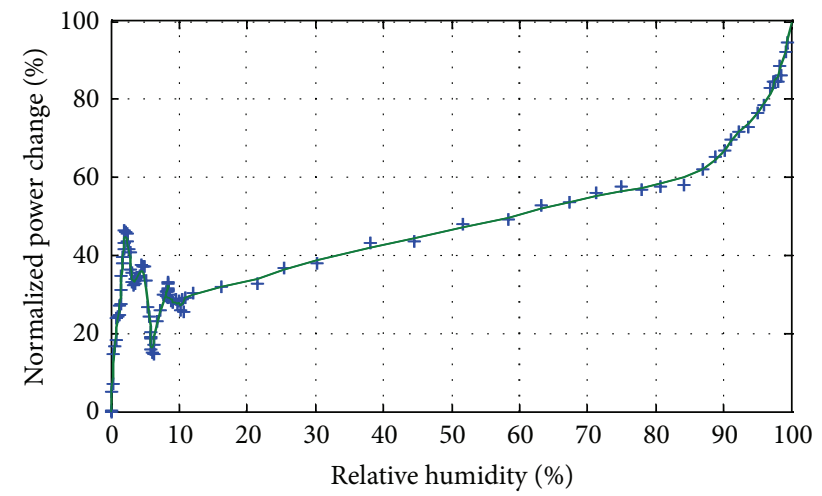

FIGURE 19: Normalized power level change as a function of relative humidity measured by the commercial sensor. Input frequency of $865 \mathrm{MHz}$ and tag-to-reader distance of $1.5 \mathrm{~m}$.

the range predicted in Figure 13. The highest peak in Figure 16 corresponds to the coupling between transmitter and receiver antennas due to the leakage signal at the second harmonic of the transmit signal generated by the nonlinearities in the power amplifier. Despite using filters at the transmitter output, the amplitude of this signal is larger than the tag reflected amplitude at the second harmonic. Modulation of the tag to exploit the receiver sensitivity is therefore needed. The other peaks around the second harmonic are due to the modulation of the tag. The received power at the modulated frequency is $30 \mathrm{~dB}$ above the noise floor; so, a good SNR is observed (about $30 \mathrm{~dB}$ ). In consequence, the uplink does not limit the read range. The tag is not powered up for distances greater than $7 \mathrm{~m}$. This result demonstrates that the main limitation is the reader-to-tag link.

Figure 17 shows the received power at the modulated frequency when the carrier frequency is swept between $830 \mathrm{MHz}$ and $875 \mathrm{MHz}$ for different relative humidity levels. It can be shown that the peak power corresponds to the frequency where the antenna is best matched. This frequency depends on the relative humidity. For relative humidity under $40-50 \%$, the change is difficult to detect as explained above because the change in the sensor capacitance is very small. Based on these results, an alternative measurement method can be used, avoiding the sweep of the oscillator frequency. The major changes in the detected power take place at a frequency of $865 \mathrm{MHz}$. The received power at the first peak of the modulated frequency is therefore used to detect the relative humidity.

Figure 18 shows the variation of the received power at $865 \mathrm{MHz}$ as a function of time. The output of the commercial sensor is also compared in Figure 18(b). The chamber is exposed to several cycles of humidity (low-high-low) by injecting dry or wet air as required. The sensor output gives values greater than $100 \%$ when the chamber is saturated with water vapour and the sensors are wet. The receiver power returns to the same value when the antenna is dry.

Figure 19 shows the normalized received power level change (variation of the received power with respect to the maximum variation of the received power) as a function of the relative humidity measured by the commercial sensor used for reference. The relationship between relative humidity and the normalized power level change is approximately linear from $10 \%$ to $90 \%$. A good correlation coefficient between the two magnitudes is found close to 0.9 . Below $10 \%$, the measurement is too noisy due to the small capacitance change of the interdigital capacitor. For values greater than $90 \%$, the capacitance changes very quickly, as can be seen in Figure 9. Figure 19 can thus be used to obtain the relative humidity from the received power measurements at the modulated frequency. A drawback of this approach is the sensibility of the DC voltage at the output of the rectenna in relation to the distance. A change in the distance varies the input RF power and therefore the rectified DC voltage, changing the oscillator frequency. Therefore, this approach can be used when the tag-to-reader distance is fixed. The problem can be solved integrating the rectifier and the oscillator including an integrated regulator similar to that used in commercial UHF tags [27]. A simple method consists in measuring the antenna detuning so that it is independent of the distance as shown in Figure 17.

\section{Conclusions}

In this study, we present an RFID humidity sensor based on a passive harmonic tag. The sensor is based on the change in the response of a patch antenna loaded with an interdigital capacitor with a capacitance that is a function of the relative humidity. The basic theory of operation of harmonic tags is introduced. A read range of $7 \mathrm{~m}$ is experimentally found, 
consistent with link budget simulations when a 2W EIRP is transmitted. This read range is limited by the powerup distance in the reader-to-tag link. A larger signal-tonoise ratio can be achieved, providing higher robustness against channel fading thanks to better receiver sensitivity of harmonic radars than in conventional backscattering RFID. Experimental results with a proof of concept using commercial components are presented. Two methods for obtaining the relative humidity of the sensor have been presented. The first is based on the measurement of the resonant frequency of the antenna by sweeping the transmitter frequency. Using this method, the response for humidities under $40-50 \%$ is poor due to the finite quality factor of the antenna and small capacitance change in this humidity range. The second method is based on the relative change in the received power level at one frequency. A good correlation coefficient is achieved with commercial sensors. Using this second method, the sensor can work at $10 \%$ to $100 \%$ of $\mathrm{RH}$ with good linearity up to $90 \%$. Further developments will focus on the size reduction of the tag antennas. In conclusion, the proofof-concept measurements open the door to harmonic tags for RFID and wireless sensor applications.

\section{Conflict of Interests}

The authors declare that there is no conflict of interests regarding the publication of this paper.

\section{Acknowledgment}

This work was supported by the Spanish Government Project TEC2011-28357-C02-01.

\section{References}

[1] K. Finkenzeller, RFID Handbook, John Wiley \& Sons, New York, NY, USA, 2nd edition, 2003.

[2] P. Pursula, M. Kiviranta, and H. Seppä, "UHF RFID reader with reflected power canceller," IEEE Microwave and Wireless Components Letters, vol. 19, no. 1, pp. 48-50, 2009.

[3] S.-C. Jung, M.-S. Kim, and Y. Yang, "A reconfigurable carrier leakage canceler for UHF RFID reader front-ends," IEEE Transactions on Circuits and Systems I: Regular Papers, vol. 58, no. 1, pp. 70-76, 2011.

[4] M. Bouthinon, J. Gavan, and F. Zadworny, "Passive microwave transponder, frequency doubler for detecting avalanche victims," in Proceedings of the 10th European Microwave Conference, pp. 579-583, September 1980.

[5] C. W. Pobanz and T. Itoh, "Microwave noncontact identification transponder using subharmonic interrogation," IEEE Transactions on Microwave Theory and Techniques, vol. 43, no. 7, pp. 1673-1679, 1995.

[6] B. G. Colpitts and G. Boiteau, "Harmonic radar transceiver design: miniature tags for insect tracking," IEEE Transactions on Antennas and Propagation, vol. 52, no. 11, pp. 2825-2832, 2004.

[7] F. Yu, K. G. Lyon, and E. C. Kan, "A novel passive RFID transponder using harmonic generation of nonlinear transmission lines," IEEE Transactions on Microwave Theory and Techniques, vol. 58, no. 12, pp. 4121-4127, 2010.
[8] S. M. Aguilar and T. M. Weller, "Tunable harmonic re-radiator for sensing applications," in Proceeding of the IEEE MTT-S International Microwave Symposium (IMS '09), pp. 1565-1568, Boston, Mass,USA, June 2009.

[9] I. T. Nassar, T. M. Weller, and J. L. Frolik, "A compact 3-D harmonic repeater for passive wireless sensing," IEEE Transactions on Microwave Theory and Techniques, vol. 60, no. 10, pp. 33093316, 2012.

[10] RECCO Rescue System, a system for locating avalanche victims, Recco AB, Liding, Sweden, http://www.recco.com/about, 2014.

[11] M. O'Neal, D. Landis, E. Rothwell, L. Kempel, and D. Reinhard, "Tracking insects with harmonic radar: a case study," American Entomologist, pp. 212-218, 2004.

[12] G. L. Lövei, I. A. N. Stringer, C. D. Devine, and M. Cartellieri, "Harmonic radar-a method using inexpensive tags to study invertebrate movement on land," New Zealand Journal of Ecology, vol. 21, no. 2, pp. 187-193, 1997.

[13] A. Singh and V. M. Lubecke, "Respiratory monitoring and clutter rejection using a CW doppler radar with passive RF tags," IEEE Sensors Journal, vol. 12, no. 3, pp. 558-565, 2012.

[14] H. Aumann, E. Kus, B. Cline, and N. W. Emanetoglu, "A lowcost harmonic radar for tracking very small tagged amphibians," in Proceedings of the IEEE International Instrumentation and Measurement Technology Conference (I2MTC '13), pp. 234-237, Minneapolis, Minn, USA, May 2013.

[15] G. Marrocco and F. Amato, "Self-sensing passive RFID: from theory to tag design and experimentation," in Proceedings of the European Microwave Conference (EuMC'09), pp. 1-4, IEEE, Rome, Italy, October 2009.

[16] J. Virtanen, L. Ukkonen, T. Björninen, A. Z. Elsherbeni, and L. Sydänheimo, "Inkjet-printed humidity sensor for passive UHF RFID systems," IEEE Transactions on Instrumentation and Measurement, vol. 60, no. 8, pp. 2768-2777, 2011.

[17] L. Yang, R. Zhang, D. Staiculescu, C. P. Wong, and M. M. Tentzeris, "A novel conformal RFID-enabled module utilizing inkjet-printed antennas and carbon nanotubes for gasdetection applications," IEEE Antennas and Wireless Propagation Letters, vol. 8, pp. 653-656, 2009.

[18] R. Vyas, V. Lakafosis, H. Lee et al., "Inkjet printed, self powered, wireless sensors for environmental, gas, and authenticationbased sensing," IEEE Sensors Journal, vol. 11, no. 12, pp. 31393152, 2011.

[19] P. Nikitin and K. Rao, "Harmonic scattering from passive UHF RFID tags," in Proceedings of the IEEE Antennas and Propagation Society International Symposium (APSURSI '09), pp. 1-4, Charleston, SC USA, June 2009.

[20] G. A. Vera, Y. Duroc, and S. Tedjini, "Redundant backscattering modulation of passive UHF RFID tags," in Proceedings of the IEEE MTT-S International Microwave Symposium Digest (IMS '13), pp. 1-3, Seattle, Wash, USA, June 2013.

[21] F. Alimenti and L. Roselli, “Theory of zero-power rfid sensors based on harmonic generation and orthogonally polarized antennas," Progress in Electromagnetics Research, vol. 134, pp. 337-357, 2013.

[22] B. Kubina, J. Romeu, C. Mandel, M. Schußler, and R. Jakoby, "Quasi-chipless wireless temperature sensor based on harmonic radar," Electronics Letters, vol. 50, no. 2, pp. 86-88, 2014.

[23] P. V. Nikitin and K. V. S. Rao, "Antennas and Propagation in UHF RFID Systems," in Proceedings of the IEEE International Conference on RFID, pp. 277-288, Las Vegas, Nev, USA, April 2008 . 
[24] S. C. M. Perera, A. G. Williamson, and G. B. Rowe, "Prediction of breakpoint distance in microcellular environments," Electronics Letters, vol. 35, no. 14, pp. 1135-1136, 1999.

[25] A. Lázaro, D. Girbau, and D. Salinas, "Radio link budgets for UHF RFID on multipath environments," IEEE Transactions on Antennas and Propagation, vol. 57, no. 4, pp. 1241-1251, 2009.

[26] I. Kwon, Y. Eo, H. Bang et al., "A single-chip CMOS transceiver for UHF mobile RHD reader," IEEE Journal of Solid-State Circuits, vol. 43, no. 3, pp. 729-738, 2008.

[27] R. Barnett, G. Balachandran, S. Lazar et al., "A passive UHF RFID transponder for EPC gen 2 with-14 dBm sensitivity in $0.13 \mu \mathrm{m}$ CMOS," in Proceedings of the IEEE International Solid-State Circuits Conference (ISSCC '07), pp. 582-623, San Francisco, Calif, USA, February 2007. 

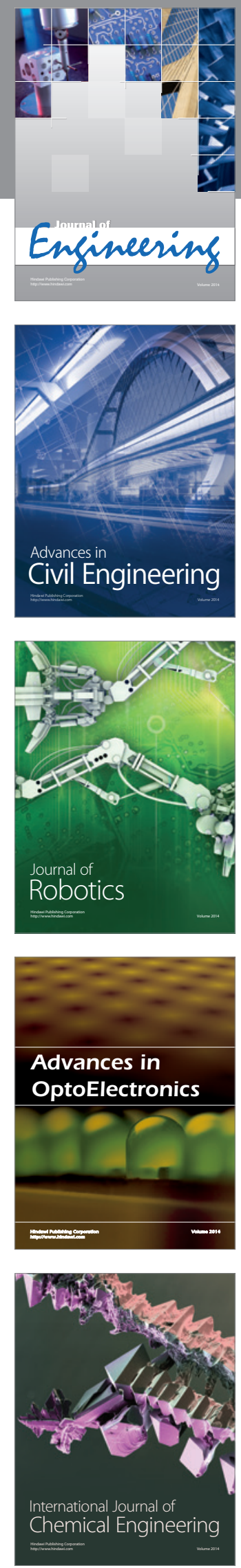

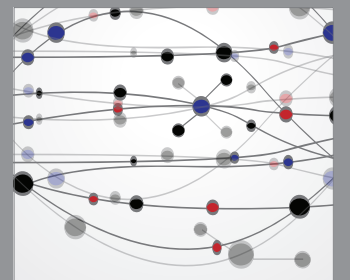

The Scientific World Journal
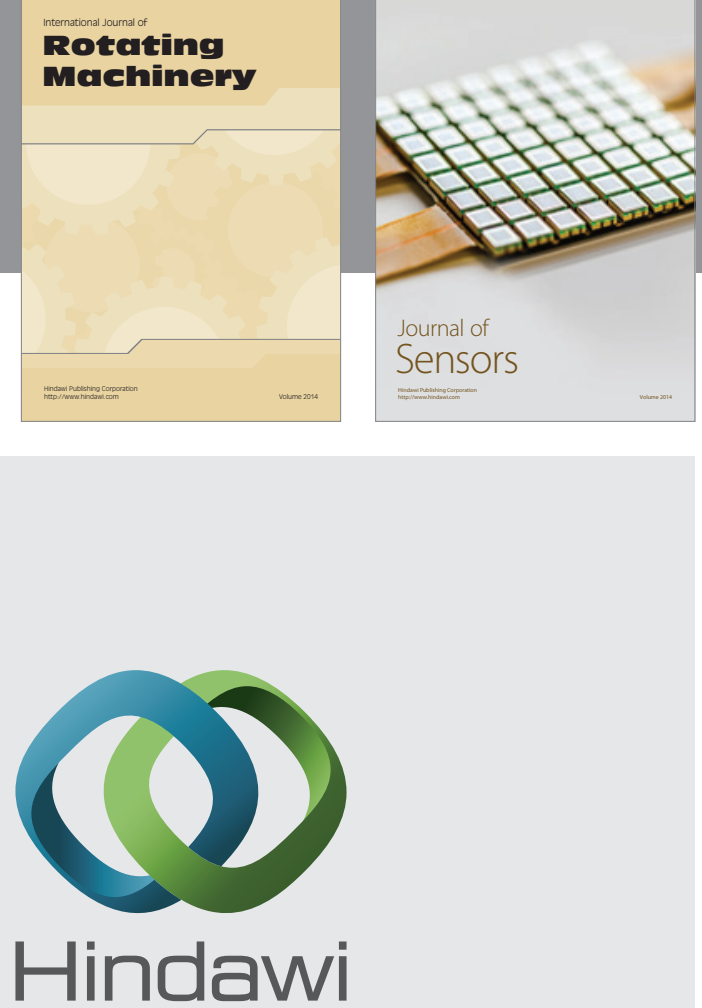

Submit your manuscripts at http://www.hindawi.com
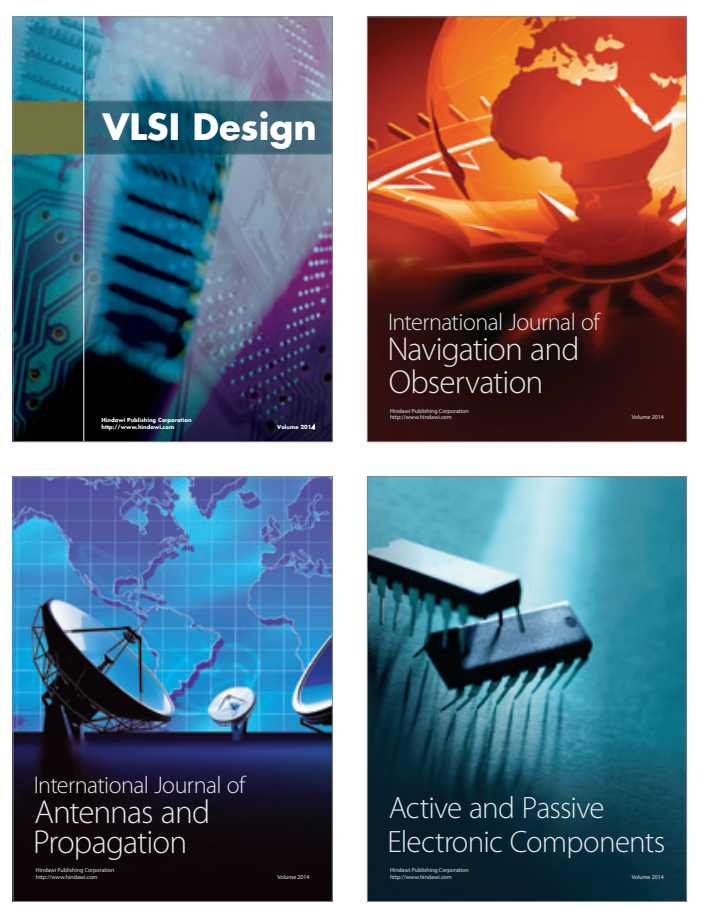
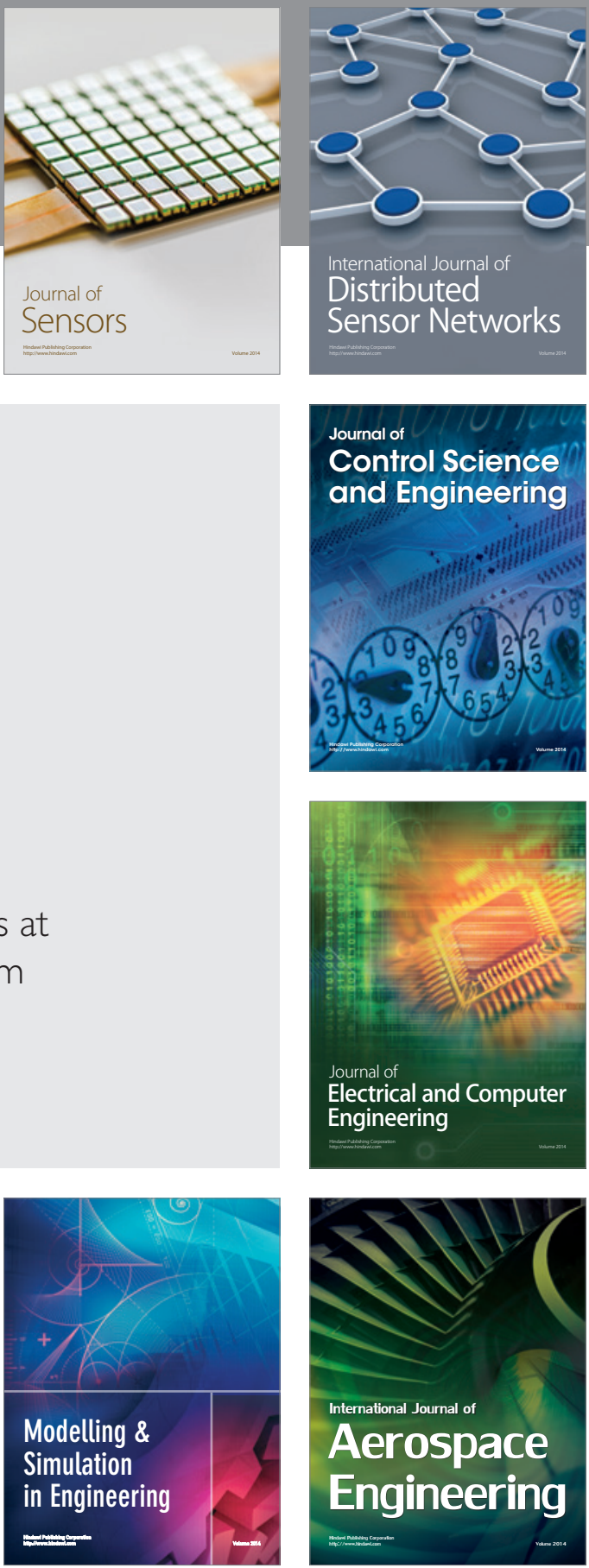

Journal of

Control Science

and Engineering
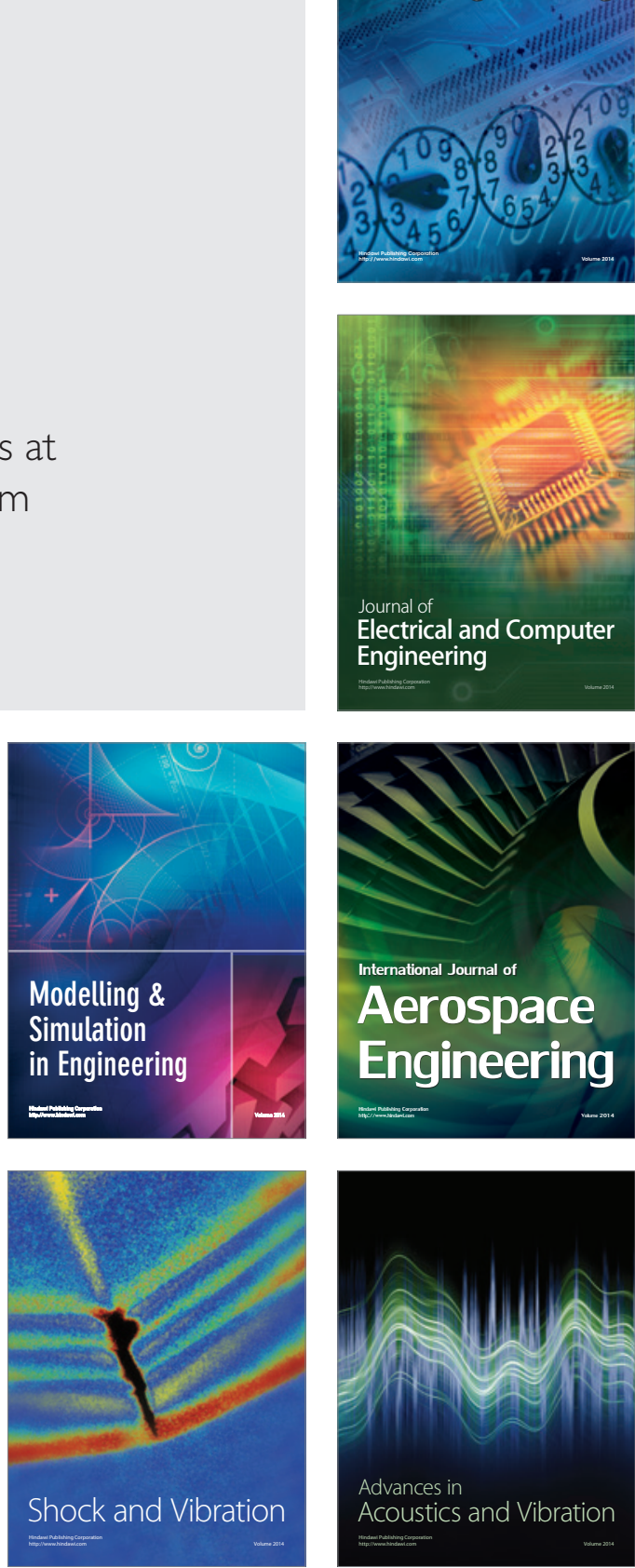